\title{
Tratamiento de la Meningitis TBC con Corticoesteroides de depósito por vía intra raquídea
}

\author{
DRES, SERGIO IBANEZ*, GUSTAVO ROSS*, VICTOR LAMA * RODOLFO BURDACH **, LUIS CUE- \\ TO ** $\mathrm{Y}$ LUIS SEMPREVIVO **.
}

Desde la iniciación de la antibioterapia de la tuberculosis se conoció el hecho que la meningitis tuberculosa, hasta entonces irresistiblemente fatal, era susceptible de curar temporaria o permanentemente con o sin secuelas.

Con el perfeccionamiento de la metodología de Ia Qujmioterapia, como la asociación de drogas útiles, conocimiento de los factores de resistencia bacteriana a los fármacos, prolongación de los tratamientos y mejor control de los enfermos, se consiguió rebajar considerablemente todos los índices epidemiológicos en la tuberculosis. Es natural que el tratamiento de la meningitis se vio favorecido por estos avances, pero presentaba aspectos propios derivados de la extrema gravedad de estos enfermos con grandes compromisos metabólicos, deshidratación, acidosis, inconsciencia, coma, descerebración, etc.

Por tales razones, parte de una quimioterapia bien llevada, debía recurrirse a elementos coadyudantes al tratamiento que favorecieran la pronta recuperación de conciencia, hidratación, alimentación y rehabilitación de estos enfermos. Se llegó así al año 1951 en que se comenzó a usar los corticoesteroides por vía general, basado en el conocimiento del efecto antiinflamatorio general de estos fármacos; sin embargo, en el caso particular de la meningitis tuberculosa, este efecto favorable de los corticoides no se veía confirmado en la clínica, sin duda por la naturaleza misma y la ubicación de las lesiones como se observaba en las necropsias de los enfermos fallecidos.

La Anatomía Patológica mostraba de manera predominante lesiones específicas endoarteriales con el correspondiente bloqueo circulatotio en importantes áreas cerebromeningeas que impedian la llegada de los medicamentos y su normal funcio-

- Departarnento de Pediatría, Hospital Calvo Mackenna.

* Departamento de Pediatría, Hospital Manuel Arriaráa. namiento. De la misma manera el grueso exudado fibrinoso de la base cerebral actuaba bloqueando toda acción terapéutica, la libre circulación del líquido céfalo-raquídeo, y además, comprimiendo las vías nerviosas. Esto explicaba por qué estos enfermos extremadamente graves, por terapéuticas tardías o erradas, muchos con recaídas de meningitis, después de una evolución tormentosa con comas prolongados o descerebración, crisis hipertérmicas o de hipertensión intracraneana, convulsiones, etc., finalmente fallecían, encontrándose en ellos que la tuberculosis estaba ya curada o en vías de serlo y que la lesión principal era de tipo mecánico y causada por la organización del exudado fíbrinoso de la base cerebral.

Ante estos hallagos quisimos orientar la terapéutica con el fin de impedir precozmente la organización del cxudado meníngeo basal, actuando localmente, por vía intrarraquídea con diferentes elementos. Así usamos, en etapas sucesivas, tripsina, estreptoquinasa y estreptodornasa, sin ningún resultado, siendo aún esta última francamente peligrosa y no recomendable. Otros habían usado anteriormente tuberculina por via raquídea sin tener tampoco éxito.

Los corticoesteroides por vía oral o parenteral habían sido ya usados por nosotros desde el año 1960 apreciándose algunos beneficios relativos. La mortalidad por meningitis tuberculosa en nuestro medio que era aproximadamente de un $40 \%$ : correspondía a la de otros autores en diferentes países. En el año 1963 comenzamos el uso de los corticoides de acción rápida (Dexametasona y $\mathrm{Be}$ tametasona) por la vía intratecal por medio de punciones raquídeas diarias, con lo que se apreciaba un rápido cambio de conciencia y el enfermo era capaz de alimentarse e hidratarse en mejor forma, cooperando así en su tratamiento.

El índice de mortalidad se vio reducido con 
esta terapia a un $30 \%$, pero adolecía del defecto de la exigencia de la administración intratecal repetida. Por esta razón en el año 1965 comenzamos la administración de corticoesterroides de depósito por vía raquídea con el objeto de obtener concentraciones altas y permanentes de drogas en el líquido céfalorraquídeo. El presente trabajo expone nuestra experiencia con estas drogas en este período de diez años.

Material y MÉTodo. Se analizan meningitis tuberculosas hospitalizadas entre los años 1962 y 1972. Se reúnen 56 casos. Se estudian los antecedentes de contacto tuberculoso, de vacunación BCG, sensibilidad tuberculínica, Bacilo de Koch en líquido céfalo-raquídeo, síntomas y mortalidad.

Todos los casos fueron tratados con drogas antituberculosas; variando su número entre 3 a 4 medicamentos. En cuanto al tratamiento corticoidal, los pacientes se reúnen en tres tipos:

A.- Prednisona oral $2 \mathrm{mgr}$. Kgr./día decrecientes por 45 días (21 casos).

B.- Prednisona oral por igual período que el anterior, más el agregado de corticoide de acción rápida, colocados intratecalmente. Se usó Dexa o Betametasona 0.1 - 0.2 mgr./ Kgr./día cada 1-2 días (9 casos).

C.-Id. tipo A más metil prednisolona acetato 1-2 mgr./kgr./día cada 5 a 7 días (26 casos). Como promedio se colocaron 2 a 3 dosis,

En cada tipo se analizan las secuelas, la mortalidad y el tiempo de normalización de la conciencia, del compromiso neurológico y del líquido céfalo-raquídeo.

La metil prednisolona acetato se presenta en frasco ampolla de 40 y $80 \mathrm{mgr}$. por $\mathrm{cm}^{3}$, en solución acuosa, lista para ser inyectada. Para su colocación se realiza una punción lumbar, la jeringa contiene la solución de este corticoide; previo a la introducción se realiza aspiración de líquido céfaloraquídeo con un movimiento rotatorio para que el medicamento se diluya convenientemente. Se inyecta efectuando maniobras de bombeo en forma lenta. No se ha producido complicación alguna en nuestra casuística.

Resultados. La edad de los pacientes oscila entre 8 meses y 13 años.

El contacto tuberculoso se conoce con certeza en 25 casos $(44 \%)$ : casi todos provienen de familiares adultos.

El PPD 2 U es positivo al inicio de la bospitalización en 38 casos $(67,8 \%)$, en los demás se hace positivo en el curso de su hospitalización.
En cuanto a la vacunación BCG se constata lo siguiente:

\begin{tabular}{|c|c|c|c|}
\hline BCG con cicatriz ...... & & ca & os $16 \%$ \\
\hline BCG sin cicatriz $\ldots \ldots$ & 7 & , & $12 \%$ \\
\hline $\operatorname{Sin} B C G \quad \ldots \quad \ldots \ldots$ & 29 & . & $51,7 \%$ \\
\hline Desconocido $\ldots . . . . . . . . . . .$. & 11 & , & $20 \%$ \\
\hline
\end{tabular}

Se aprecia que los vacunados BCG, enferman menos que los no vacunados.

El cultivo del bacilo de Koch en líquido cé falo-raquídeo es positivo en 17 casos (30,3\%).

Los síntomas previos al diagnóstico se manifiestan entre 2 y 90 días y consisten en decaimiento, fiebre, cambios de carácter, compromiso general, etc. que se presentan en el $100 \%$ de nuestros pacientes.

Además existe compromiso meníngeo y neurológico en el siguiente orden:

\begin{tabular}{|c|c|}
\hline gros meníngeos & \\
\hline Somnolencia y obnubilación & 28 \\
\hline Inconsciencia $\ldots \ldots \ldots \ldots$ & 18 \\
\hline Hemiplegia o Herniparesia .... .... .... & 16 \\
\hline $\begin{array}{llllllll}\text { Convulsiones } & \ldots & \ldots & \ldots & \ldots & \ldots & \ldots & \ldots\end{array}$ & 14 \\
\hline 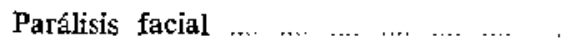 & 12 \\
\hline $\begin{array}{ccccccc}\text { Estrabismo } & \ldots & \ldots & \ldots & \ldots & \ldots & \ldots\end{array} \ldots$ & 8 \\
\hline
\end{tabular}

El fondo de ojo, examen practicado de rutina en todos los niños, muestra en 21 hechos positivos:

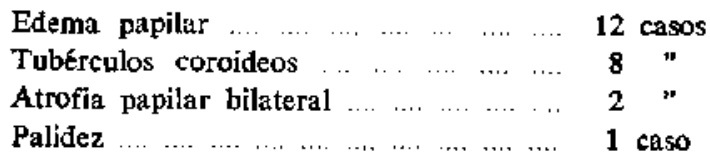

Concomitante a la meningitis tuberculosa en 29 pacientes, se encuentra tuberculosis pulmonar activa $(51,7 \%)$; mal de Pott; tuberculosis renal y suprarrenal en un caso cada uno.

En 26 casos no se demuestra foco tuberculoso extrameníngeo activo $(46,4 \%)$.

De los 56 pacientes, 24 quedan con secuelas al alta $(43 \%)$. Estas consisten en:

\begin{tabular}{|c|c|}
\hline esias & 8 casos \\
\hline Alteraciones conductuales $\ldots \ldots \ldots$ & \\
\hline Retardo psíquico & 3 \\
\hline Compromiso facial & 3 \\
\hline $\begin{array}{lllll}\cdots & \ldots & \ldots & \ldots & \ldots\end{array}$ & 3 \\
\hline
\end{tabular}

Fallecen 8 casos $(14,3 \%)$. De estos en 4 se hace el diagnóstico tardíamente. En otros dos el diagnóstico es precoz pero por su extrenta gravedad fallecen antes de los 5 días de tratamiento.

De los dos restantes fallece uno por colapso vascular provocado por insuficiencia suprarrenal y el otro por bidrocefalia operada. Estos dos últimos casos son recaídas. 
Los pacientes a su ingreso se clasifican en tres grupos; según el Medical Research Council; United Kingdom:

Grupo I. Signos y síntomas de compromiso general, tales como fiebre, inapetencia, decaimiento, cambios de carácter, etc.

Grupo II. Síntomas y signos de hipertensión intracraneana y meníngeos.

Grupo III. Los anteriores, más parálisis y coma.

Según esto, todos los pacientes que recibieron tratamiento intratecal, sea de efecto rápido o de depósito fueron grupo III.

Respecto al tratamiento y evolución del grupo I y II, en los cuales existe compromiso leve, en ellos el tratamiento se realizó precozmente, en todos se obtuvo mejoría completa.

Sin embargo, el grupo III es el que nos da la mortalidad y nos deja secuelas importantes. En este grupo es donde usamos prednisolona acetato. Anclizaremos con detalle la evolución de estos enfermos en cuanto a la normalización del líquido céfajo-raquídeo, recuperación de conciencia y restablecimiento reurológico.

DIAS DE NORMALIZACION EN MENINGITIS TUBERCULOSA GRUPO III

\begin{tabular}{crrcc}
\hline & LCR & Conciencia & Neurolugicos \\
\hline Tratamiento Tipo A & 1.21 & 80 & 122 \\
$"$ & Tipo B & 138 & 24 & 27 \\
$"$ & Tipo C & 68 & 25 & 70 \\
\hline
\end{tabular}

Es evidente que los pacientes que recibieron Metil prednisolona-acetato, tiepen una mejoría más rápida respecto a los tres parámetros arriba anotados, en comparación con aquellos que recibieron solamente corticoterapia oral.

La secuelas y la mortalidad son más bajos, también, en los pacientes que recibieron corticoides de depósito de acción lenta, como se observa en la tabla siguiente:

SECUELAS GRUPO $/ 1 /$

\begin{tabular}{cccccc}
\hline & & & Casos & Secuelus & $\%$ \\
\hline Tratamiento Tipo & A & 8 & 5 & 62 \\
$"$ & Tipo & B & 6 & 3 & 50 \\
$"$ & Tipo & C & 25 & 8 & 32 \\
\hline
\end{tabular}

MORTALIDAD Y TIPOS DE TRATAMIENTO EN GRUPO HI

\begin{tabular}{cccc}
\hline Tipo & Casos & Mortalidad & $\%$ \\
\hline A & 21 & 4 & 19 \\
B & 9 & 3 & 33 \\
C & 26 & 1 & 3,8 \\
\hline
\end{tabular}

Cmentario. Desde que se inició el tratamiento con corticoides en la meningitis tuberculosa, se observó una menor tasa de mortalidad, recuperación más rápida del paciente y disminución de las secuelas. Sin embargo los resultados, no eran muy promisorios en aquellos que llegaban con un proceso avanzado, sea por consulta o diagnóstico tardío. En estos niños, al fallecer se encontraba una organización importante del exudado fibrinoso de la base cerebral, con todas las consecuencias que ello significaba. Por esta razón, se resolvió usar terapéuticas intrarraquídeas, quedándonos en forma definitiva con los corticoides intratecales de acción lenta. (acetato de Metil prednisolona).

Al comparar los resultados obtenidos en los tres grupos de meningitis tuberculosa graves (grupo III), se observa que las secuelas y la mortalidad son menores, y que la normalización del líquido céfalo-raquídeo, de la conciencia y del compromiso neurológico son más rápidos en los pacientes tratados con corticoides intratecal de acción lenta.

No se hace referencia a este tipo de tratamiento en la literatura consultada.

\section{RESUMEN}

Los autores revisan 56 casos de meningitis tuberculosa, tratados en la forma habitual, más el agregado de corticoides por vía intrarraquídea, sean éstos de acción rápida o de depósito. En cada grupo de tratamiento se analizan los casos graves, en cuanto a su mortalidad, secuelas y normalización de diferentes parámetros.

Se concluye que la administración intratecal do Metil prednisolona-acetato es francamente ventajosa para la pronta recuperación de los pacientes.

Se obtuvo una disminución de la mortalidad del $19 \%$ at 3,8\%. Las secuelas bajaron del $62 \%$ al $32 \%$ y se recuperaron en menos tiempo el LGR, la conciencia y el compromiso neurológico.

\section{Bibliografía}

1.-Kendig. E.: Disorders of the Respiratory Tract in Children. Philadelphia, W. B. Saunders Company. 1967. 
2.--Nelson, W., Vaughan, Vicior. Tratado de Pediatría. Sexta edición, 614-615, 1971.

3.-Gellis. S., Kagan. B. Current Pediatric Theraphy-4. $845-846,1970$.

4.-- Robbins. S. Tratado de patología II edición 1116 $1117,1962$.

5.-Voliaree. B., and Corpe. R. The influence of cotticosteroid Hormones in the treatment of tuberculous meningitis in Negrces. The American Review of Respiratory diseases. Vol. 81, 539-545, April, 1960.

6.-Choremis. C.. Putpadalos. C., Gargoulas. A., and Drosos, $C$, intrathecal Hydrocortisone in the treatment of tuberculous Meningitis. The journal of Pediatrics. Vol. 50, 138-J44. Febrary, 1957.

7.-Wasz Hockert. Ole, Modern treatment and late Prognosis of Tuberculous meningitis. Acta Pediáttica 51. Suppl 141: 93-102. October 1962.

8.- Shane, S. J., and Riley. G. Tuberculous Mepingitis, The New England journal of Medicine. Vol. 249. 829-834. November, 1953.

9.-González. Oscar, Palomino, Carmen, Irarrizaval, Miguel, y Kraljevic. Roque. Tratamiento de la Meningitis tuberculosa con glucocorticoides. Revista Médica de Chile. Vol. 95. 762, 767, 1967.
10.—Maffioletti. F, Paiacios E. Merchak, N, y Burdach. $k$. Meningitis tuberculosa tratamiento esterroidal. Pediatría. Vol. 4. 292-296 Oct.-Nov.-Dic. 1961.

11.-Todd. Mc Loren and Neville Joseph. The sequelee of tuberculous meningitis. Arch Dis Chidh Vol. 39. 213-225, 1964.

12,-Bulkeley. Winifred. Tuberculous Meningitis treated with ACTH and Isoniazid. British Medical journal Vol. 21. 1127-1129. Nov., 1953.

13,-Kerr, Jotm. Treatment of Tuberculous Meningitis. British Medical journal. Vol. 211130 - Nov., 1953.

14.-Johuson. R., Furstenberg N., Patterson. R., Schoch H. and Dovey $W$. Corticotropin and adrenal steroids as adjucts to the treatment of tuberculous Meningitis, Annals of Internal Medicine. Vol, 46. No 2. 316-331, 1957.

15.-Toole. R., Thomton. G, Mukheriee. M., and Hath. L. Dexamethasona in tuberculons Meningitis. Annale of Internal Medicine. Vol. 70, No 1, 1969.

16.-Ghosh. Shanti, Seshardri. R., and Jain. C. Evaluation of Corticoesteroids in treatment of Tuberculous Meningitis, Arch of Disease in Childhood. Vol. 46. 51-54, 1971.

17.-Miller, F, Seal. R., and Taylor. M. Tuberculosis in Children-Little, Brown and company. 395-422, 1963. 